\title{
TITLE: Disconnection of Open Coronal Magnetic Structures
}

\author{
AUthor(S): D.J. McComas, J.L. Phillips, A.J. Hundhausen, J.T. Burkepile
}

submitted to: Proceedings of the Solar Wind 7 sonference, Goslar, Germany, 16-20 September 1991

\section{DISCLAIMER}

This report was prepared as an account of werk sponsored by an agency of the United States Government. Neither the United States Government nor any agency thereof, nor any of their employees, makes any warranty, express or implied, or assumes any legal liability or responsibility for the accuracy, completeness, or usefulness of any information, apparatus, product, or process disclosed, or represents that its use would not infringe privately owned rights. Reference herein to any specific commercial product, process, or service by trade name, trademark, manufacturer, or otherwise does not necessarily constitute or imply its endorsement, recommeidation, or favoring by the United States Government or any agency thereof. The views and opinions of authors expressed herein do not necessarily state or reflect those of the United States Government or any agency thereof. 
DISCONNECTION OF OPEN CORONAL MAGNETIC STRUCTURES

D.J. McComas,* J.L. Phillips,* A.J. Hundhausen,** and J.T. Burkepile**

*Space Plasma Physics Group, MS-D438, Los Alamos National Laboratory, Los

Alamos, NM 87545, USA, ** High Altitude Observatory,

Boulder, CO, USA

\begin{abstract}
We have examined the Solar Maximum Mission coronagraph/polarimeter observations for evidence of magnetic disconnection of previously open magnetic structures and a number of likely examples have been found. Probable coronal disconnections typically appear as pinching off of helmet streamers followed by the release and outward acceleration of a large $U$ or V-shaped structures. The observed sequence of events is consistent with reconnection across the heliospheric current sheet between previously open magnetic field regions, and the creation of a detached magnetic structure which is open to interplanetary space at both ends. Sunward of the reconnection point, coronal disconnection events would return previously open magnetic flux to the Sun as closed field arches. Here we 1) describe one clear disconnection event ( 1 June 1989); 2) examine the results of a limited survey of disconnection events; and 3) discuss the potential importance of coronal disconnections for maintaining open magnetic flux in interplanetary space.
\end{abstract}

\title{
INTRODUCTION
}

Coronal structures and transients are commonly observed in coronagraph observations of the Sun. Such observations measure the line of sight integrated Thomson scattered white light which is proportional to electron density. Owing to the high electrical conductivity of the coronal plasma, which ties the plasma to the local magnetic field, bright structures in coronagraph observations are generally interpreted as magnetic structures populated with higher density plasma. 
The typical coronal structure is characterized by a combination of "open" coronal holes which have roughly radial magnetic fields extending out into interplanetary space and low coronal densities and "closed" arch and loop structures wrich have higher coronal densities. So-called helmet streamers are regions where oppositely directed open fields are juxtaposed above closed field arches. These streamers map out into the heliospheric c'Irrent sheet $/ 1$. In addition to long lasting open and closed structures, the solar corona is routinely disturbed by coronal transient events. The most notable subset of coronal transients are called coronal mass ejections or CMEs /2,3/. Classical CMEs, which display loop-like structures rising out of the solar corona in sequences of coronagraph pictures, are generally interpreted as the eruption of previously closed field regions on the Sun. While there are other definitions of the term CME, we will use it to mean these eruptions of new flux and plasma in contrast to a broader range of coronal transients.

Most CMEs appear to leave long lasting "legs" which slowly fade into coronal holes. As such, CMEs appear to open previously magnetically closed regions of the Sun into long lasting open field regions. One long standing question of coronal physics is that if CMEs do open new magnetic flux into interplanetary space. why doesn't the amount of open flux, and hence interplanetary magnetic field (IMF) magnitude, grow without bound? The general question of the maintenance and evolution of open magnetic flux in interplanetary space has been discussed previously /4/ and is examined elsewhere in this proceedings $/ 5 /$.

One important aspect of the maintenance of the solar magnetic flux in interplanetary space may be that at least some of the flux is returning to the Sun elsewhere in the corona; helmet streamers provide an ideal location for reconnection in the corona which would lead to the return of magnetic flux to the Sun $/ 4,5 /$. Such reconnection would also lead to the release of " $U$ " or "V" shaped structures which would be open to the outer heliosphere at both ends. One sequence of Solar Maximum Mission (SMM) coronagraph observations was recently published showing a U-shaped structure being detached and released from a helmet streamer on 27 June $1988 / 4 /$. The primary purpose of this study is to extend the examination of the SMM coronagraph data for further evidence of such reconnection and the release of $U$-shaped magnetic structures.

\section{OBSERVATIONS}

Figure 1 displays a sequence of four white light chronograph pictures for a coronal transient event which occurred on June 1, 1989. The 6:32 UT panel shows a coronal streamer (straight up in this figure). By 11:10 UT a clear separation is forming at approximately two solar radii (arrow) between the lower, sunward pointing arches and apparently disconnected $U$-shaped magnetic flux. This separation continues to develop (14:16 UT panel), and finally, by 17:05, the bulk of the outward pointing flux has risen, leaving a bundle of closed field arches tied to the Sun in its wake. It is worth noting that the sequence of images 
displayed here is only a small subset of those taken over this day; the other images show various intermediate configurations and a rapid stepping through of the images (as was shown at the Solar Wind 7 meeting) displays the disconnection event far better than the four panels of Figure 1.

Differenced images of the central interval of this event have also been made utilizing a pre-event image from 9:38 UT. Four of these differenced images are displayed in Figure 2. Lighter gray regions indicate an increase in electron density compared to the preevent image while darker gray indicates a deficit at later times. Clearly the region of detached plasma and flux grows and rises with time while an ever increasing deficit is observed along at least the left flank of this region. This configuration is at least qualitatively consistent with $\mathrm{X}$-line type reconnection where plasma and open magnetic flux are brought into the reconnection region from the sides while reconnected flux is ejected sunward into closed field arches and antisunward into $U$-shaped structures.

The general survey of the SMM coronagraph data /6/ identified some suggestion of a disconnected structure in only $3 \%$ of all coronal transient events. However, this survey was not generally conducted with an emphasis on disconnection, and we were aware of at least several examples of apparent disconnections which were not identified in the initial survey. In order to asses the frequency of occurrence of coronal disconnection events, we carefully reexamined an interval in which no magnetic disconnections were noted in the initial survey. In particular, we reexamined the first 52 coronal transient events identified in 1988 (1/1/88 through 3/26/88). While there were no large, obvious events in this interval, we found six ( $11 \%$ ) that displayed some reasonable evidence for reconnection in sequences of several frames and another 13 (23\%) that showed a "U" or "V" shaped structure in a single coronagraph frame. Since the appearance of such structures can also be caused by projection effects of bright streamers, single frame observations cannot be unambiguously interpreted.

\section{DISCUSSION}

Coronal disconnection events are not as obvious in sequences of coronagraph images as are large CMEs. However, there are a number of clear examples of coronal disconnections in the SMM coronagraph data set; in our reexamination of 52 corcnal transient events we found reasonable evidence for disconnection in 6 events $(>10 \%)$. In spite of the complications inherent in interpreting coronagraph observations, we conclude that disconnection events are more common in the solar corona than has been generally appreciated. By and large the coronal disconnection events that we have examined have not appeared as the release of plasmoid-type CMEs with both the eruption of new flux from the Sun and its subsequent disconnection of this flux. Rather, we find coronal disconnection events generally occurring over helmet streamers on previously open magnetic fields. 
Figure 3 schematically displays a time sequence for reconnection across a helmet streamer. The top panel shows a normal helmet streamer configuration; in middle panel reconnection has begun. Such reconnection could begin on already open field lines or on the closed, innermost arches of the helmet structure and continue through to the open fields. The plasmoid loop in the center of the disconnected flux (second panel of Figure 3) schematically indicates thai this particular disconnection started on closed field lines. In either case if reconnection occurs sufficiently close to the Sun that the newly created arches are bound to the Sun and are not freely expanding. flux is effectively returned to the Sun whenever reconnection occurs on previously open field lines. In the bottom panel, reconnection has continued on the open field lines across the helmet streamer, and a region of " $U$ " or "V" shaped magnetic field has been released while newly closed arches has been returned to the Sun.

We believe that the combination of CMEs, which remain at least partially attached to the Sun. opening new flux into interplanetary space $/ 5 /$, and coronal disconnection events returning previously open flux to the Sun $/ 4$ / is a fundamental process in the evolution and maintenance of open magnetic flux in interplanetary space. Even though we have shown here that coronal disconnections occur more frequently that was previously appreciated. they are clearly less common that CMEs. Two possible explanations for this inequality are 1) CMEs having a "flux rope" type geometry in which only a fraction of the CME flux is newly opening /5/ and 2) magnetic reconnection routinely occurring across helmet streamers at a slow rate but only unusually large and impulsive disconnection events being observable remotely with coronagraphs.

Work at the National Center for Atmospheric Research, sponsored by the National Science Foundation, was supported by NASA contracts S55989 and S55989A. Work at Los Alamos National Laboratory was conducted under the auspices of the United States Department of Energy.

\section{REFERENCES}

1. J.T. Gosling, G. Borrini, J.R. Asbridge, S.J. Bame, W.C. Feldman, and R.T. Hansen, Coronal streamers in the solar wind at $1 \mathrm{AU}$, J. Geophys. Res., 86, 5438 (1981)

2. J.T. Gosling, E. Hildner, R.M. MacQueen, R.H. Munro, A.I. Poland, ani C.L. Ross, Mass ejections from the Sun: a view from Skylab, J. Geophys. Res., 79, 4581 (1974)

3. A.J. Hundhausen, The origin and propagation of coronal mass ejections, Proc.Sixth Int. Solar Wind Conf., ed. V.J. Pizzo, T. Holzer, and D.G. Sime, NC.AR/TN-306+Proc 1988, p. 181.

4. D.J. McComas, J.L. Phillips, A.J. Hundhausen, and J.T. Burkepile, Observations of disconnection of open coronal magnetic structures, Geophys. Res. Lett., 18, 73 (1991) 
5. D.J. McComas, J.T. Gosling, and J.L. Phillips, Regulation of the Interplanetary Magnetic Flux, this issue.

6. O.C. St. Cyr and J.T. Burkepile. A catalogue of mass ejections observed by the Solar Maximum Mission coronagraph. NCAR Technical Note, NCAR/TN-352+STR (1990)

7. O.C. St. Cyr and A.J. Hundhausen. On the interpretation of "halo" coronal mass ejections, Proc.Sixth Int. Solar Wind Conf., ed. V.J. Pizzo, T. Holzer, and D.G. Sime, NCAR/TN-306+Proc 1988, p. 253. 


\section{Figure Captions}

Fig. 1. Sequence of SMM coronagraph observations of coronal detachment on 1 June 1989.

Fig. 2. Images of the roronal detachment differenced from a preevent image at 9:38 UT. Lighter (darker) gray indicates greater (lesser) intensity at later times compared to the preevent image.

Fig. 3. Time sequence of schematic diagrams showing a coronal disconnection event. Once the initially open magnetic fields on either side of the helmet streamer are reconnecting, magnetic flux is returned to the Sun and a U-shapeu magnetic field lines are released. 


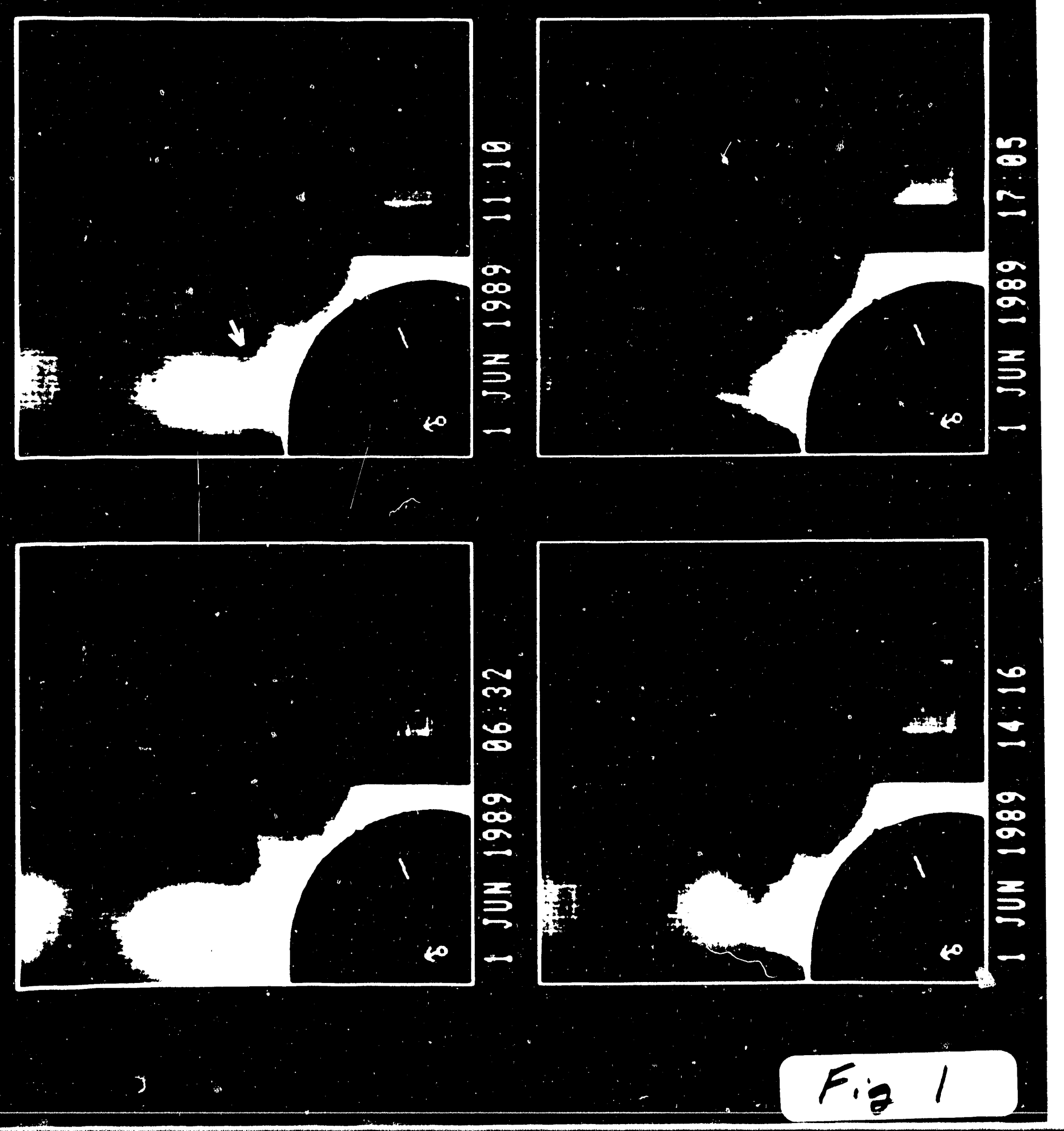



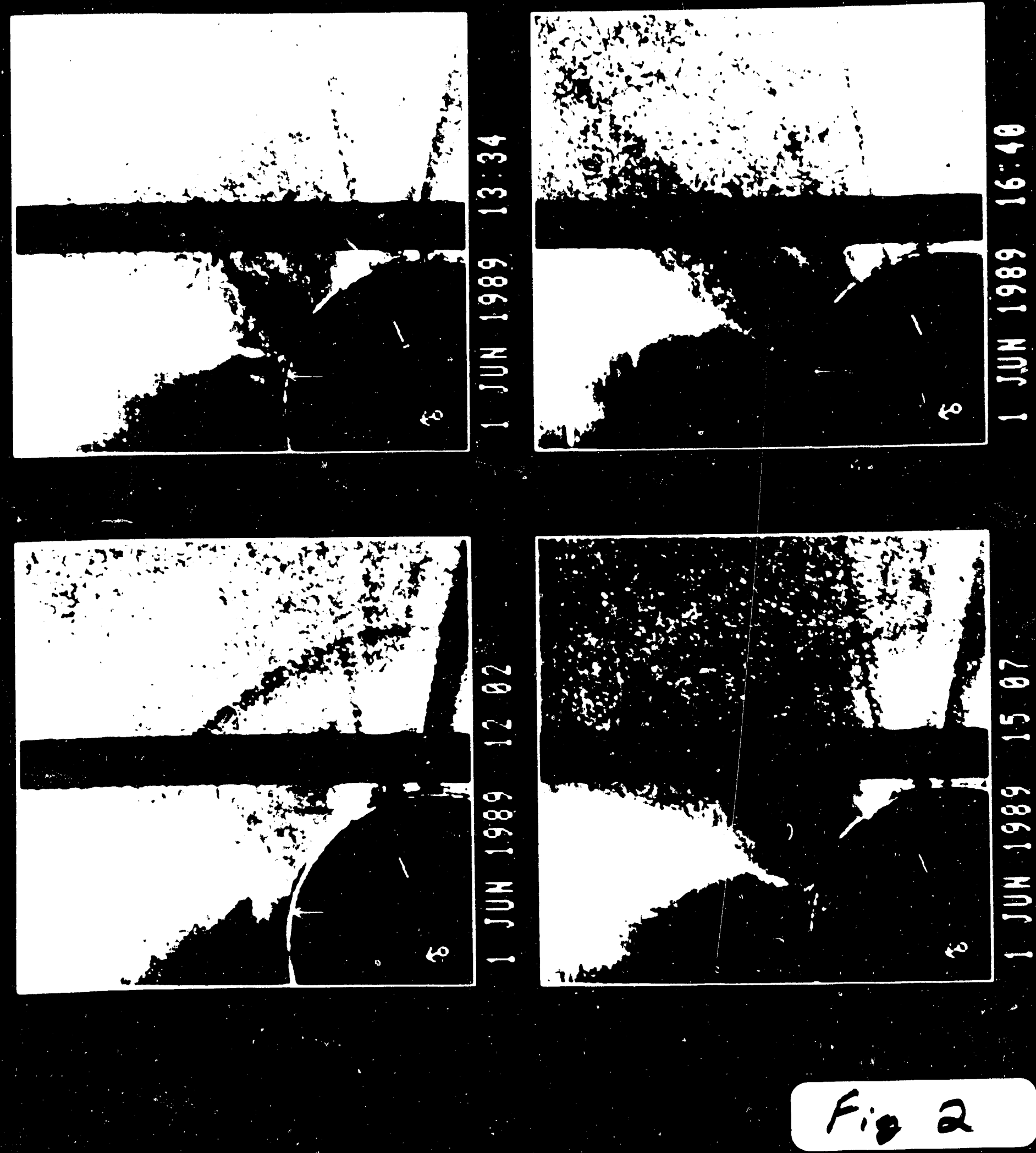

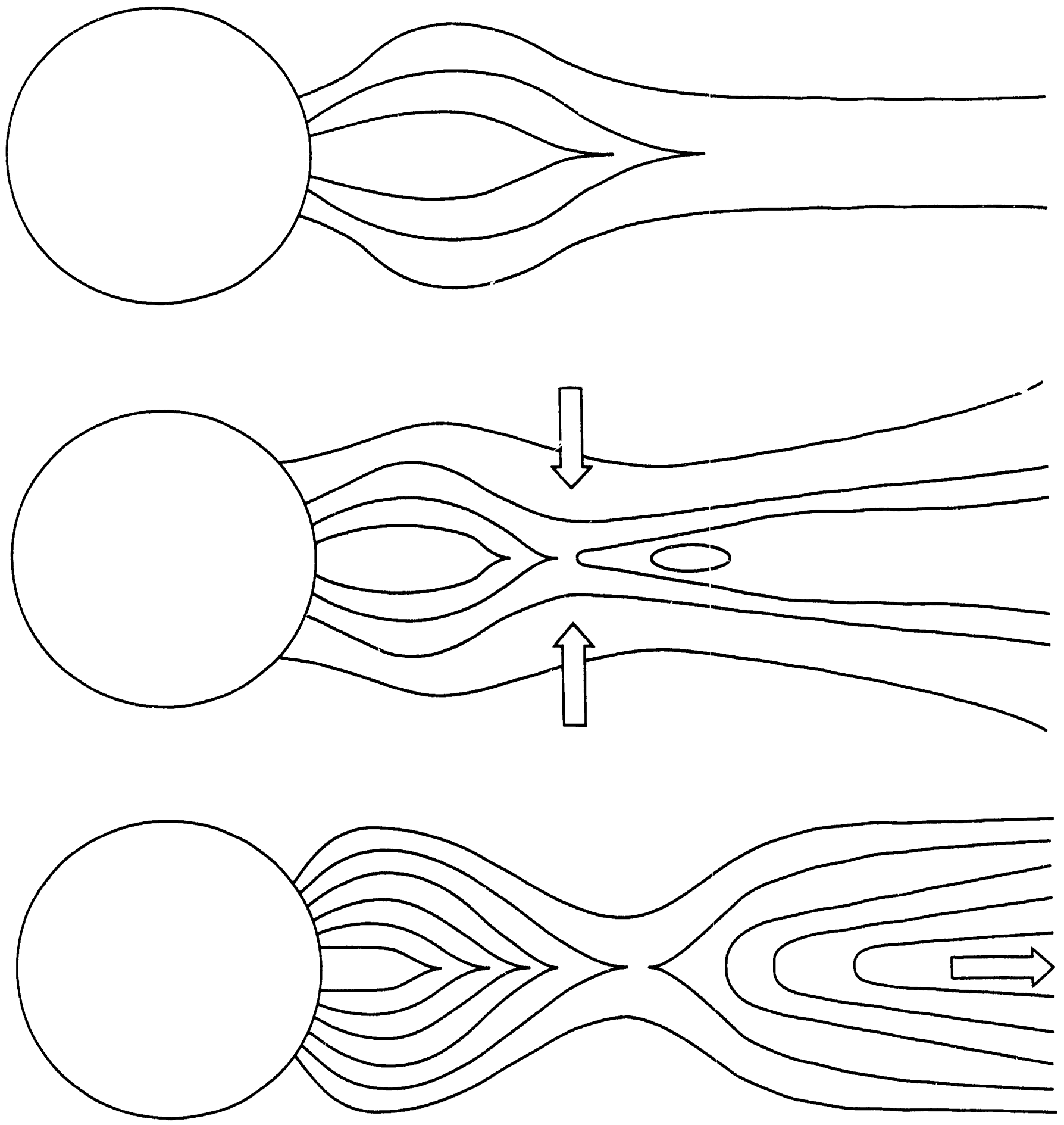

Fig. 13 

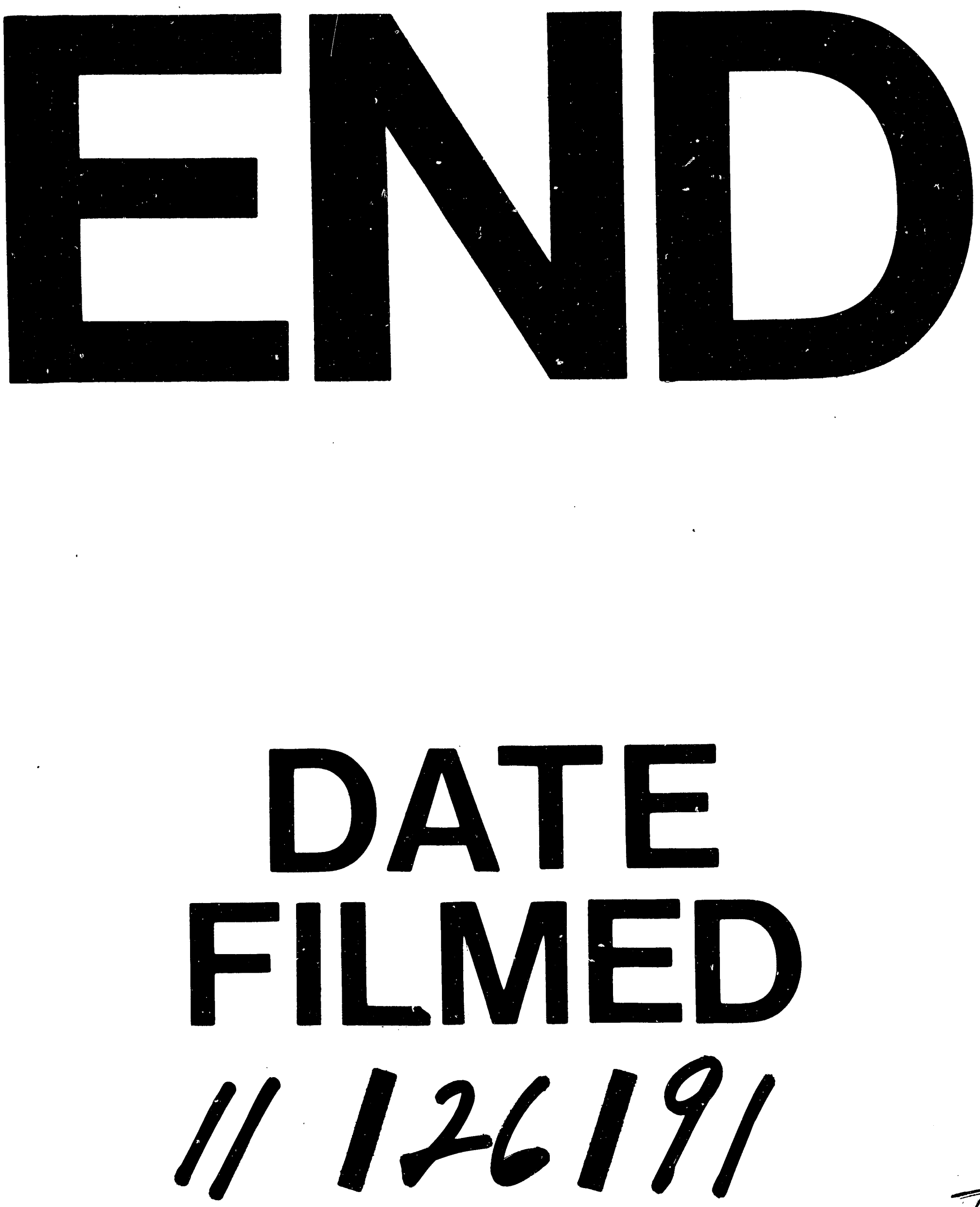

$\overline{1}$ 
